\title{
Transfert de la littérature acadienne en Pologne : bilan d'une expérience traductologique et didactique
}

\author{
Teresa Tomaszkiewicz \\ Université Adam Mickiewicz
}

\section{Préliminaires}

Dans cet article, nous aimerions faire le bilan d'une recherche entamée en automne 2005 à l'Institut de philologie romane de l'Université Adam Mickiewicz, à Poznań, en Pologne. L'objectif principal de ce projet est la diffusion et la promotion en Pologne de la littérature, langue et culture des communautés minoritaires du Canada français, notamment acadiennes (Claude LeBoutillier, France Daigle, Charles Pelletier, etc.), franco-ontariennes (Jean Marc Dalpé, Daniel Poliquin, Robert Dickson), fransaskoises (David Baudemont) et franco-manitobaines (Gabrielle Roy, J. R. Léveillé, Simone Chaput). Pour le réaliser, nous avons décidé, tout d'abord, de traduire en polonais des œuvres choisies des auteurs mentionnés ci-dessus afin de présenter au lecteur polonais « les littératures de l'exiguïté » (Paré 2002) du Canada français, c'est-à-dire, les œuvres littéraires en provenance de l'extérieur du Québec.

Nous avons prévu les étapes suivantes de la réalisation de ce projet :

1) La traduction des œuvres acadiennes choisies (les autres entreront dans la suite du projet) en polonais, tout d'abord par les étudiants en maîtrise. Cette partie a été réalisée entre octobre 2005 et décembre 2006;

2) La préparation des maîtrises dont l'objet fut l'analyse des difficultés traductologiques ayant surgi lors de la préparation des traductions en question (la réalisation de cette partie a été terminée en juin 2007);

3) La publication d'une anthologie de passages traduits avec un commentaire scientifique;

4) Le suivi de la réception de ces traductions littéraires dans la presse populaire et littéraire, ainsi que dans les publications scientifiques (nous envisageons l'organisation de la lecture de ces textes auprès des étudiants de la philologie polonaise suivie d'une enquête sur la réception);

5) Des réflexions littéraires et théoriques sur la littérature acadienne, ainsi que sur sa traduction.

\section{Modalités de l'expérience}

Étant directrice de philologie romane, j'ai participé à une rencontre organisée à Sèvres en 2005, où nous avons débattu de l'idée de la propagation de la francophonie dans les centres universitaires. En Pologne, la majorité des programmes d'enseignement $\mathrm{du}$ français au niveau universitaire sont organisés autour de la 
littérature et de la civilisation française. Rares sont les tentatives d'introduire dans la formation les œuvres ou les données socioculturelles et historiques portant sur d'autres pays francophones. J'ai pensé que l'on pourrait remplir cette lacune par un enseignement sur le Canada francophone. C'était donc l'idée qui a donné naissance au programme que je présenterai dans les pages qui suivent.

Six étudiantes se sont présentées au séminaire de maîtrise en septembre 2005. Nous leur avons proposé de travailler sur la traduction des œuvres acadiennes choisies. D'emblée, on peut se poser la question: "Qu'est-ce que cela veut dire : choisies?» Qui opère ce choix? Qui décide des valeurs que l'on souhaiterait transmettre dans une nouvelle zone réceptrice? Comment faire rapprocher les valeurs d'une culture qui semble inconnue dans le contexte d'arrivée?

\subsection{Le choix des textes à traduire}

Nous pensons que la description des étapes de notre démarche est importante pour démontrer le rôle des milieux universitaires dans les échanges interculturels à l'échelle internationale et dans la diffusion des littératures aussi bien mineures que majeures. En outre, il ne faut pas sous-estimer le rôle que peut jouer le hasard quant au choix des œuvres littéraires fournies par la culture source, puis retenues pour traduction par la culture réceptrice. Des spécialistes polonais et canadiens nous ont fourni certains renseignements quant aux auteurs dignes d'intérêt, et nous avons fait les recherches dans les bibliothèques en Pologne. Aucun des auteurs suggérés n'y figurait, et aucun des auteurs n'avait été traduit en polonais ${ }^{1}$.

\footnotetext{
${ }^{1}$ En 2005, nous avons invité Denise Merkle de l’Université de Moncton, université qui dessert la communauté acadienne, à venir à Poznań pour présenter les conférences sur la littérature acadienne et la traduction littéraire. Nous avons conclu la visite sur l'entente qu'elle allait nous faire parvenir une sélection d'œuvres littéraires acadiennes pour traduction éventuelle par les étudiants inscrits au $2^{\mathrm{e}}$ cycle à $l^{\prime}$ Institut de philologie romane.
} 
Onze titres nous sont parvenus du Canada ${ }^{2}$, et ils étaient généralement conformes aux contraintes que nous avions précisées : une variété de genres afin de plaire aux goûts différents, le niveau linguistique ou stylistique pas trop difficile étant donné que les étudiantes n'allaient pas être des spécialistes de la traduction littéraire ${ }^{3}$, et pas trop longs non plus (c'est-à-dire, faisables dans le cadre d'un cours d'un an).

Avant de choisir le texte à traduire, il a fallu que chaque étudiante lise les onze œuvres mises à notre disposition. Les étudiantes ont retenu les titres suivants : L'Acadien reprend son pays de Claude LeBoutillier, Petites difficultés d'existence de France Daigle, L'oasis de Charles Pelletier, Les Portes-tournantes de Jacques Savoie, Disgrâce de l'humanité : essai sur la torture de Serge Patrice Thibodeau et Terre sur mer

${ }^{2}$ Voici les références :

1) L'Acadien reprend son pays, Claude LeBoutillier, Moncton, Éditions d'Acadie, 1977.

2) Petites difficultés d'existence, France Daigle, Montréal, Boréal, 2002.

3) L'oasis, Charles Pelletier, Moncton, Éditions d'Acadie, 1993.

4) Les Portes-tournantes, Jacques Savoie, Montréal, Boréal, 1990.

5) Disgrâce de l'humanité : essai sur la torture, Serge Patrice Thibodeau, Montréal, VLB éditeur, 1999.

6) Terre sur mer, Mario Thériault, Moncton, Éditions Perce Neige, 1997.

7) La Sangouine, Antoine Maillet, Montréal, Bibliothèque québécoise, 2004.

8) Miniatures, Herménégilde Chiasson, Moncton, Éditions Perce-Neige, 1995.

9) Infarctus parmi les piétons, Christian Roy, Moncton, Éditions Perce-Neige, 2000.

10) L'Ennemi que je connaissais, Martin Pître, Moncton, Éditions Perce-Neige, 1995.

11) Pas Pire, France Daigle, Montréal, Éditions Boréal, 2001.

${ }^{3}$ En concevant le projet en question j'ai pris en compte les prémisses différentes que celles de Jerzy Jarniewicz (2002). Celui-ci distingue deux types de traducteurs littéraires : 1) les traducteurs-ambassadeurs [notre traduction] qui ne cherchent pas à établir leurs propres systèmes de valeurs, mais traduisent les œuvres considérées comme fondamentales par la culture qui les a produites pour informer la culture d'arrivée de ce qui est canonique ; 2 ) les traducteurs-législateurs qui introduisent dans la culture d'arrivée les textes qui ne sont pas nécessairement les plus populaires chez eux mais qui, d'après le traducteur, peuvent entrer dans un dialogue créateur avec la sienne, tout en présentant de nouvelles formes littéraires et linguistiques, voire un nouveau système de valeurs. À cette vision du traducteur qui est un connaisseur de la littérature et de la culture de départ s'oppose la figure du traducteur-artisan [notre traduction] qui produit les traductions commandées par des maisons d'édition. Cellesci développent une politique éditoriale soumise aux lois du marché. Finalement, dans les systèmes totalitaires la politique éditoriale est soumise aux décisions prises par les dirigeants. Elżbieta Skibińska (2006) constate à ce propos : «Dans la Pologne des années 1946-1960 cette manipulation [du choix de textes à traduire] se fait selon les normes acceptées et légitimées par les cercles politiques et culturels dominants, assignant à la traduction différentes fonctions ; ainsi, parmi les descriptions du monde créées par les romanciers français, sont sélectionnées celles qui servent les besoins non pas de la littérature d'accueil mais des dirigeants du parti au pouvoir » (140). 
de Mario Thériault. Comme c'était leur première année du $2^{\mathrm{e}}$ cycle, les étudiantes qui ont pris part au projet n'avaient pas participé aux conférences organisées en 2005. Elles n'avaient donc aucune idée de l'Acadie, de son histoire ni de sa littérature, ces éléments n'entrant pas dans les programmes de formation.

\subsection{Post-traduction}

Une fois les traductions terminées, j'ai demandé aux participantes d'analyser le travail effectué en fonction de trois points de vue :

1) celui du premier lecteur qui juge un texte qu'il vient de lire, sans avoir effectué une recherche dans les sources ;

2) celui du traducteur qui est obligé de comprendre toutes les nuances au niveau du contenu et de la forme et de trouver des équivalences traductologiques ;

3) celui du récepteur de la traduction.

Pour unifier cette réflexion, j'ai proposé une enquête comportant une vingtaine de questions.

\section{Résultats de l'enquête}

\subsection{I ère étape : Première réception}

Tout d'abord, les œuvres choisies sont variées du point de vue du genre et des thématiques. Les étudiantes n'ont pas retenu un recueil de poésie ayant opté pour les textes écrits en prose : trois romans, deux nouvelles, un récit de voyage et un essai. En outre, le degré de leur ancrage respectif dans la culture de départ est variable. L'Acadien reprend son pays et Petites difficultés d'existence sont les romans les plus attachés à la culture acadienne. Les Portes-tournantes l'est aussi, mais d'une manière moins explicite. Le recueil de nouvelles Terre sur mer, tout en évoquant des endroits déterminés, traite des questions plus générales, voire universelles, c'est-à-dire des questions humaines. Par contre, le récit de voyage L'Oasis évoque une tierce culture (l'Inde), et l'auteur de Disgrâce de l'humanité: essai sur la torture, tout en étant un personnage très actif et visible dans la culture acadienne locale, s'occupe des problèmes humains à l'échelle internationale. C'est pourquoi le jugement de ces œuvres est aussi variable.

Nous avons essayé de cerner la première réception de chacun des textes à l'aide des questions suivantes :

A. Qu'est-ce qui vous a intéressé le plus dans le texte original ?

B. Quelles étaient les premières barrières de compréhension (les barrières linguistiques, thématiques, socioculturelles, idéologiques) ? Quels étaient les éléments qui, pendant la première lecture, vous ont paru « exotiques »?

C. Quels éléments du contenu vous paraissaient-ils universels, à savoir compréhensibles pour chaque personne, indépendamment de sa culture? Quels autres éléments pouvaient-ils interpeler immédiatement les Polonais? 


\subsection{A. Qu'est-ce qui vous a intéressé le plus dans le texte original ?}

La réponse à cette question me paraît très importante car c'est la première impression $\mathrm{du}$ traducteur qui dirigera en quelque sorte son projet de traduction dans lequel il tâchera de préserver les qualités détectées initialement pour les transmettre au récepteur d'arrivée (voir Annexe 1, qui présente de façon très sommaire la réponse des étudiantes à la première question). Quant au choix des textes à traduire, différents éléments peuvent retenir l'attention du lecteur/traducteur et éveiller le désir de les transférer dans un autre milieu.

\subsection{B. Quelles étaient les barrières de compréhension?}

Évidemment, déjà à la première lecture, il y avait des éléments difficiles à comprendre et des éléments «exotiques». Faute de place nous n'allons pas développer l'idée de la traduction de ces « exotismes ». Rappelons seulement que les décisions concernant l'ingérence du traducteur dans l'original pour expliquer au récepteur étranger ce qui peut lui paraître inconnu doivent être pondérées. Les réflexions théoriques à ce propos se laissent diviser en deux groupes. Les plus connues et «classiques » (Vinay et Darbelnet 1958 : 52-53; Catford 1965: 164-168; Taber et Nida 1971; Newmark 1988: 135) présentent les arguments presque identiques: ne pas présenter d'obstacle à la compréhension, éviter la « couleur locale ", considérée comme une surtraduction. À cette attitude s'oppose une autre préconisée par Berman (1985), Lécrivain (1998), Lewicki (2000), entre autres, qui se prononcent en faveur de la présence de l'Autre dans le texte traduit. Dans ce qui suit nous tâcherons de démontrer qu'aucune position extrême n'est souhaitable lorsqu'il s'agit de traduire les éléments exotiques au risque de bloquer complètement la compréhension en voulant, par exemple, «faire l'épreuve de l'étranger » selon la formule de Berman. (Voir l'Annexe II, qui présente les premières barrières de compréhension, voire les éléments « exotiques ».)

\subsection{Quelles sont les valeurs universelles ?}

Évidemment, malgré les différentes barrières de compréhension, les textes à traduire communiquent des valeurs universelles, ainsi que celles qui pourraient toucher tout particulièrement les lecteurs polonais. (L'Annexe III fournit quelques exemples donnés par les étudiantes.)

\subsection{II $^{\text {ème }}$ étape : traduction}

La deuxième série de questions servait à réfléchir sur le processus de traduction.

1. Énumérez les plus grandes difficultés traductologiques: a) au niveau linguistique (vocabulaire, notions, structures grammaticales, noms propres, style-registre, figures de style, etc.); b) au niveau des relations sociales présentées; c) au niveau du contenu (l'histoire ancrée dans un contexte 
historique, culturel et idéologique déterminé, et inscrite dans les événements sociaux spécifiques).

2. Précisez des interventions du traducteur (développements définitionnels, explications, notes du traducteur, recherche des équivalences, adaptation, omissions): a) Dans quels cas les consultations avec les étudiantes canadiennes $^{4}$, les professeurs ou d'autres spécialistes se sont-elles avérées nécessaires pour comprendre le texte et trouver des solutions traductologiques ? b) Quelles sont d'autres sources d'informations que vous avez utilisées pour comprendre les éléments historiques, sociaux et linguistiques ? c) Qu'est-ce qui vous paraît encore problématique?

\subsubsection{Difficultés traductologiques}

Il est clair que les difficultés traductologiques variaient d'un texte à l'autre. Par ailleurs, beaucoup de ces problèmes ne concernent pas uniquement la littérature acadienne, mais aussi d'autres types de textes rédigés en français, dont les textes littéraires en général. Mais bon nombre de ces difficultés résultaient du fait que les traductrices n'avaient tout simplement pas beaucoup d'expérience en traduction. Il n'empêche que certaines observations concernaient la spécificité de la littérature acadienne. En voici des exemples regroupés de manière synthétique.

Le premier problème, et peut-être le plus important, concerne Petites difficultés d'existence et l'emploi du vernaculaire du sud-est du Nouveau-Brunswick, le chiac. D'après Catherine Leclerc (2005), le chiac «[...] despite its sometimes anglicized phonology, lexicon and even syntax is a dialect of French. [...] Indeed, from a sociological perspective, Chiac connotes a specific Francophone identity, as only Acadians speak Chiac» (161). Il en résulte que la présence de cette variante linguistique est très importante du point de vue de l'identité des Acadiens du sud-est $\mathrm{du}$ Nouveau-Brunswick. Plus loin Leclerc ajoute : «Writing in Chiac (and, to a lesser extend, writing about Chiac) is to be part of a battle against ideological stigmatisation, under the influence of Parisian literature, of the literary use of vernacular language in all literatures written in French» (2005: 163). Nous avons donc affaire à un projet idéologique qu'il fallait transmettre au lecteur polonais. Mais la chose paraissait très difficile. La tension ressentie par la traductrice polonaise n'est pas nouvelle. Par exemple, Antoine Berman (1984) ainsi que Jacques Derrida (1985) exprimaient leur scepticisme devant la possibilité de traduire adéquatement un texte plurilingue, hybride ou écrit en vernaculaire. Il faut noter aussi que la présence des

\footnotetext{
${ }^{4}$ Grâce à une subvention du CRSH, chaque étudiante polonaise avait une correspondante à l'Université de Moncton (étudiante inscrite au baccalauréat de traduction) qui essayait, dans la mesure du possible, de fournir par courriel des réponses aux questions concernant les données culturelles, historiques, linguistiques, etc. Pour de plus amples informations sur la recherche subventionnée par le CRSH, voir http://francais.concordia.ca/conf07/MERKLE_KLIMKIEWICZ.pdf.
} 
anglicismes dans les dialogues paraît relativement problématique dans la traduction polonaise.

Nous avons essayé d'introduire quelques expressions dialectales polonaises dans le texte, mais elles semblaient trop marquées stylistiquement et tout de suite faisaient penser à une région concrète de la Pologne, au lieu d'évoquer une variante spécifique du français. Mais le problème ne s'arrêtait pas là. Bon nombre d'anglicismes compris par les Acadiens restent obscurs pour les Polonais. En plus, le polonais est une langue flexionnelle qui accepte mal des substantifs empruntés à l'anglais au milieu des phrases où on ne peut pas les décliner. Finalement, nous avons opté pour les solutions proposées par Robert Majzels et décrites par Leclerc. Dans sa traduction de Petites difficultés d'existence en anglais, Majzels « ha[d] to find a balance between ethnography and assimilation » (2005: 176). Il en résulte que les dialogues de France Daigle en polonais sont marqués par la langue parlée, avec certains éléments dialectaux et certains éléments de l'argot des jeunes, tout en comportant des anglicismes que les Polonais peuvent comprendre ${ }^{5}$.

Le deuxième texte difficile, mais d'un autre point de vue, est $L^{\prime}$ Acadien reprend son pays. Tout d'abord, il est important de signaler le décalage temporel entre la parution de ce texte (1977) qui parle de l'avenir (1987) et la réalisation de la traduction en 2007. Ainsi, l'original étant considéré comme roman d'anticipation l'est moins en traduction. Par ailleurs, cette œuvre fictive est profondément ancrée dans l'histoire de l'Acadie: le lecteur est confronté aux détails de la réalité et aux évènements véridiques mélangés aux événements inventés. Dans ce roman nous retrouvons les noms propres réels : les villes de Moncton, Pocesudie et GrandeAnse; les rivières Restigouche, Nipisiguit et Peticodiac; ainsi que quelques noms inventés tels que Bathurst Square Power (compagnie anglaise), la compagnie Alibaba, la cellule Jones de Moncton et l'Association des Louveteaux d'Acadie.

Il est difficile d'abord de comprendre et ensuite de rendre les allusions à l'histoire de l'Acadie, p. ex., la fondation de l'école normale française pour les Acadiens, la transformation du Nouveau-Brunswick en province bilingue, la fondation de l'Université de Moncton en 1963, la fondation de Halifax par les Britanniques, le voyage de Jacques Cartier à Gaspé, les manifestations de 1971 à Kouchibougouac et l'évènement le plus important dans l'histoire des Acadiens, le Grand Dérangement, sans oublier l'épopée nationale Évangéline. Si l'on transfère ces faits et mythes historiques uniquement au niveau dénotatif, on leur enlève toute la charge affective inhérente et les rend insaisissables pour le lecteur polonais.

\footnotetext{
${ }^{5}$ En ce qui concerne la traduction de la langue parlée, on peut se référer à Tomaszkiewicz
} (2007). 
Mais finalement, ce qui apparaissait le plus important dans ce roman, c'était les métaphores filées ${ }^{6}$ constituant les champs sémantiques des termes décrivant les Américains ou les anglophones (négativement) et les Acadiens francophones (positivement). Il importe de souligner que la reconstruction de la vision binaire véhiculée par le roman est fondamentale pour la réception de l'œuvre. Une reconstruction précise de certaines oppositions antonymiques: puissance impuissance, soumission - autonomie, justice - injustice paraît peut-être moins difficile que le transfert précis de certains faux synonymes fréquents : détruire - polluer, petitminoritaire, peur - inquiétude. À ces séries de mots s'ajoutent les adjectifs évaluatifs axiologiques dénotant les deux pôles opposés :

\begin{tabular}{|l|l|}
\hline Monde acadien & monde anglophone \\
\hline $\begin{array}{l}\text {...un ordre social où les valeurs humaines ne } \\
\text { seraient pas dominées par la notion de profit } \\
(24)\end{array}$ & ...une voracité insatiable (21) \\
\hline ...les Acadiens éparpillés un peu partout (48) & $\begin{array}{l}\text {...la consommation éhontée et le chewing- } \\
\text { gum à saveur de pétrole (24) }\end{array}$ \\
\hline $\begin{array}{l}\text {...ils se sentaient désarmés et impuissants dans } \\
\text { cet effort pour bâtir leur pays (29) }\end{array}$ & $\begin{array}{l}\text {...une civilisation aux poumons d'acier et au } \\
\text { cœur métallique (23) }\end{array}$ \\
\hline
\end{tabular}

Cette liste peut être complétée par les substantifs qui, à plusieurs endroits du texte, décrivent le sentiment d'infériorité éprouvée par les Acadiens: frustration, soumission, fatalisme, échec. Ils s'opposent à la vision des anglophones: monstres, voracité, supergrand. Ajoutons encore des verbes affectifs comme échouer et réussir (69).

Afin de choisir une stratégie traductologique appropriée, le traducteur doit répondre au préalable aux questions suivantes :

- Veut-il transférer la culture étrangère ou les informations sur cette culture?

- Cherche-t-il à trouver les parallélismes entre les évènements et les valeurs acadiens et polonais ou cherche-t-il à montrer l'Autre acadien comme exotique?

- Est-ce que le but de la traduction est de communiquer de façon impartiale la réalité de la vie de l'Autre, de l'Acadien, ou est-ce qu'il essaie de créer les attitudes affectives positives par rapport à la cause défendue dans l'œuvre?

- Cherche-t-il à transférer des valeurs universelles de l'œuvre ou opte-t-il plutôt pour souligner son exotisme?

En répondant à ces questions (voir Annexe IV), on voit tout de suite que beaucoup de valeurs exprimées dans $L^{\prime}$ Acadien reprend son pays sont comparables aux

\footnotetext{
${ }^{6}$ La métaphore filée ou continuée est « un trope dont le noyau de signifié se répartit sur plusieurs signifiants tropiques qui apportent chacun une indication secondaire ou détaillée au sens fondamental » Mazaleyrat/Molinié (1989 : 79).
} 
valeurs exprimées dans la littérature polonaise engagée de différentes époques : la volonté de préserver sa langue, la lutte pour l'indépendance, la domination culturelle et politique, le sentiment d'infériorité, le sentiment de victimisation, la conviction que l'on est le peuple martyre qui a toujours besoin de revendiquer ses droits. Ainsi, il est de toute pertinence de souligner que cette œuvre tellement ancrée dans la culture de départ, mais qui ne fait pourtant plus partie du canon de la littéraire acadienne, peut devenir un texte proche aux récepteurs polonais grâce aux questions traitées dans le roman.

Quant à la traductrice de Disgrâce de l'Humanité: essai sur la torture, elle a rencontré un autre type de problèmes d'ordre lexical et terminologique : l'emploi $d^{\prime}$ un certain vocabulaire, du registre et des noms propres. Tout d'abord les termes du domaine de la torture proviennent de plusieurs langues, à savoir de l'anglais (p. ex., shellacking, breaking the news, to break under the torture, working on), de l'espagnol ( $\mathrm{p}$. ex., pau de arara, teléfono, campana), de l'hébreu (p. ex., tiltul, shabeh). Certaines appellations des tortures n'ont pas d'équivalents polonais (grenouille, banane, etc.). Les termes juridiques apparaissent dans les citations tirées des publications documentaires internationales. La traduction officielle de certaines d'entre elles n'existe pas en polonais, et c'est la raison pour laquelle leur traduction est devenue parfois problématique. Après avoir retrouvé tous les équivalents en polonais, et après avoir traduit l'ouvrage, un autre problème s'est présenté.

Est-ce que la traductrice était capable de montrer et transférer tous les sentiments et tous les détails importants du point de vue de l'auteur ? Est-ce que le phénomène de torture décrit dans le texte avec tant de délicatesse et de finesse est reproduit avec précision par la traductrice ? Serge Patrice Thibodeau était militant de l'Amnistie internationale, ce qui lui a donné la possibilité de côtoyer les victimes et les survivants de la torture. Faute d'une telle expérience, la traductrice a trouvé sa tâche d'autant plus difficile.

Dans le récit Oasis, c'est la présence de la culture de l'Inde qui représente une difficulté majeure. Mais celle-ci n'était pas la seule. Le problème que la traductrice ne pouvait pas résoudre, c'était de comprendre l'expérience que fait un Canadien moyen de cette culture. À quel point ce contact interculturel serait-il comparable avec celui d'un Polonais moyen? Est-ce que les expériences et les impressions de l'auteur sont aussi exotiques pour le récepteur canadien que pour le récepteur polonais ? La réponse à ces questions était importante car elle pouvait contribuer à une surtraduction éventuelle.

Le roman Les Portes-tournantes ne véhiculait pas, quant à lui, de problèmes traductologiques ancrés dans les faits de culture. Au niveau linguistique, les problèmes rejoignaient ceux qu'on peut rencontrer généralement dans les différents 
textes français, à savoir les temps verbaux, les valeurs de « on », le tutoiement et le vouvoiement, et les onomatopées. Pourtant un élément a retenu notre attention : un style narratif qui comptait cinq narrateurs. Il a fallu recréer le même effet stylistique.

La moins problématique était la traduction de deux nouvelles du volume Terre sur mer : «Clémence » et «La communion des mélomanes ». À part quelques comparaisons difficiles à traduire, p. ex., "le non-dit règne sur le village comme l'orgue sur un match de hockey " (13), "Le marketing transpirait de moi comme la sueur du corps des habitants des tropiques"(17), "Mes vrais parents avaient dépassé l'âge des voitures sportives et des extravagances yé-yé, comme les premières cassettes huit pistes, incorporées aux radios » (16) et quelques éléments de la réalité trop étrangers, p. ex., " passer à la commission », qui veut dire acheter de l'alcool, le texte ne présentait pas trop de difficultés. Il n'empêche que, à nouveau, la traduction de certains mots anglais s'est avérée problématique : "Ça va décoiffer, c'est plus hard » (55), "a groove était bonne » (52). Comme nous avons déjà expliqué, il était difficile de les incorporer dans un texte polonais.

\subsubsection{Solutions traductologiques et les consultations avec les natifs}

En ce qui concerne les solutions traductologiques, elles paraissent semblables à celles $q^{\prime}$ 'on peut trouver dans d'autres types de traduction. Par contre, ce qui peut être intéressant, c'est de voir comment la consultation avec les étudiants ou d'autres spécialistes de la culture de départ pouvait aider les traductrices. (À l'Annexe IV, nous présentons les problèmes que les traductrices ont discutés avec leurs correspondantes.) Les étudiantes n'ont évidemment pas pu résoudre tous les problèmes de traduction et de transfert culturel.

\subsection{III $^{\text {ème }}$ étape : réception de la traduction}

Voici les questions posées sur la réception de chaque traduction par un lecteur polonais :

- Quels sont les éléments que le lecteur polonais pourrait-il ne pas comprendre?

- Est-ce que le manque de connaissances de la réalité acadienne de la part du/par le lecteur polonais moyen pourrait créer des barrières de compréhension?

- Si oui, pourquoi ?

- Est-ce que malgré l'éloignement important entre les deux cultures, ces œuvres peuvent intéresser le lecteur polonais?

- Quelles sont les valeurs générales que le lecteur peut tirer de sa lecture?

Encore une fois nous regroupons les réponses les plus significatives sous forme de tableau synoptique (voir l'Annexe V).

\section{La réflexion théorique}


Comme nous avons mentionné au début de ce rapport de recherche, chaque apprentie traductrice s'est servie de ses expériences de traduction pour alimenter une réflexion théorique qui allait être à la base de son travail de maîtrise. Au mois de juin 2007, six mémoires de maîtrise ont été soutenus ${ }^{7}$.

\section{Conclusion}

Ce rapport de recherche n'a aucune prétention d'exhaustivité, d'autant plus qu'il ne présente qu'une partie du projet à long terme. Il n'empêche que l'expérience menée pendant deux ans permet déjà de tirer quelques conclusions provisoires :

1. En réfléchissant sur l'implantation d'une littérature minoritaire dans un milieu récepteur éloigné il faut bien veiller sur «le choix des ouvrages». Ce choix dépend entre autres de la visée traductologique: informationnelle («traduction-ambassadrice» [notre traduction], cf. Jarniewicz 2002) ou recherchant les similitudes des valeurs («traduction-législation» [notre traduction], cf. Jarniewicz 2002).

2. Ce travail demande la création d'un réseau $\mathrm{d}^{\prime}$ « ambassadeurs » de la cause. Les traducteurs peuvent jouer un tel rôle.

3. Un réseau de contacts établis favorise la prise de conscience de l'Autre.

4. L'intraduisibilité n'est pas forcement fonction de la méconnaissance de certains « réalia » (cf. Tomaszkiewicz 2001, 2002), mais beaucoup plus de la méconnaissance d'une certaine charge affective et du jeu de connotations qui sont ancrées dans la culture de départ ${ }^{8}$.

5. Une bonne analyse de cette charge affective permet souvent de retrouver le même réseau de jeu connotatif dans la culture d'arrivée, ce qui facilite le transfert.

${ }^{7}$ Voici les auteures, suivies du titre de leur mémoire respectif :

1) Agnieszka Kowalska : «Traduction commentée du roman L'Acadien reprend son pays de Claude LeBouthillier »

2) Katarzyna Lach : "Transferts de la réalité culturelle et linguistique acadienne sur l'exemple du roman Petites difficultés d'existence de France Daigle »

3) Natalia Marczuk : «Traduction commentée du roman Oasis, Itinéraire de Delhi à Bombay de Charles Pelletier»

4) Anna Szybiak : « La traduction de différents styles de narration sur l'exemple des Portes tournantes de Jacques Savoie»

5) Ewa Wiechcińska : «L'analyse de la traduction de l'œuvre de Serge Patrice Thibodeau La Disgrâce de l'Humanité : essai sur la torture»

6) Anna Wojciechowska : «Traduction de la langue parlée sur l'exemple des nouvelles de Mario Thériault Clémence et La Communion des mélomanes »

${ }^{8}$ À propos de la traduction de la charge affective, voir A. Żuchelkowska, 2005. 
6. La réflexion théorique qui suit une formation pratique des traducteurs facilite leur prise de conscience et leur fournit une solide base pour le travail professionnel. 


\section{BIBLIOGRAPHIE}

BERMAN, Antoine (1984), L'épreuve de l'étranger: Culture et traduction dans l'Allemagne romantique, Paris, Gallimard.

BERMAN, Antoine (1985), «La traduction comme épreuve de l'étranger », Texte 4, 67-81.

CATFORD, J. C. (1965), A Linguistic Theory of Translation, London, Oxford University Press.

DERRIDA, Jacques (1985), «Appendix: Des tours de Babel », dans Graham Joseph (dir.), Difference in Translation, Itaca and London, Cornell University Press, 209-248.

JARANIEWICZ, Jerzy (2002), « Tłumacz jako twórca kanonu », dans Roman Lewicki (dir.), Przekład-Jezzyk-Kultura, Lublin, Wydawnictwo UMCS, 35-42.

KLIMKIEWICZ, Aurelia et Denise MERKLE (2007), " The Internet and Nomadism: Literary Translation from (Acadian) French to Polish», site de l'Université Concordia, Département d'études françaises [En ligne] http://francais.concordia.ca/conf07/MERKLE_KLIMKIEWICZ.pdf

LECLERC, Catherine (2005), «Between French and English, Between Ethnography and Assimilation: Strategies for Translating Moncton's Acadian Vernacular», TTR XVIII/2, 161-192.

LÉCRIVAIN, Claudine (1998), "Europe, traduction et spécificités culturelles », dans Michel Ballard (dir.) Europe et traduction, Arras/Ottawa, Artois Presses Université/Les Presses de l'Université d'Ottawa, 345-358.

LEWICKI, Roman (2000), Obcość w odbiorze przekładu, Lublin, Wydawnictwo Uniwersytetu Marii Curie-Skłodowskiej.

MAZALEYRAT, Jean et Georges MOLINIÉ (1989), Vocabulaire de la stylistique, Paris, PUF.

NEWMARK, Paul (1981), Approaches to Translation, Londres, Pergamon.

NEWMARK, Paul (1988), A Textbook of Translation, New York, Prentice Hall.

NIDA, Eugene (1964), Towards the Science of Translation, Leiden, Brill.

PARÉ, François (1992), Les littératures d'exiguïté, Ottawa, Le Nordir.

SKIBIŃSKA, Elżbieta (2006), «La traduction au service de l'idéologie: Liste des lectures françaises en polonais dans les années 1946-1960», dans Michaela Wolf (dir.), Übersetzen-Translating-Traduire: Towards a «Social Turn»?, Berlin/Wien, LIT Verlag, 131-141.

TABER, Charles R. et Eugene NIDA (1971), La traduction: théorie et méthode, s.l., Alliance Biblique Universelle.

TOMASZKIEWICZ, Teresa (2001), « Transfert des références culturelles dans les sous-titres filmiques », dans Yves Gambier et Henrik Gottlieb (dirs.), (Multi)Media Translation, Amsterdam/ Philadelphia, John Benjamins Publishing Company, 237249.

TOMASZKIEWICZ, Teresa (2002), « Traduction des textes déjà censurés », TTR XV/2, 171-190. 
TOMASZKIEWICZ, Teresa (2007), «Transfert des différents registres de la langue parlée », dans Corinne Wecksteen et Ahmed El Kaladi (dirs.), La traductologie dans tous ses états, Arras, Artois Presses Université, 161-176.

VINAY, Jean et Jean-Paul DARBELNET (1958), Stylistique comparée du français et de l'anglais, Paris, Didier.

ŻUCHELKOWSKA, Alicja (2005). « Transfert de la charge affective sur l'exemple de la traduction en français des oeuvres polonaises choisies ». Thèse de doctorat inédite (Institut de philologie romane, Université Adam Mickiewicz). 


\section{Annexe I : L'intérêt du texte de départ}

\begin{tabular}{|l|l|}
\hline L'Acadien reprend son pays & $\begin{array}{l}{ }^{9} \text { Histoire de l'Acadie, lutte pour l'identité nationale, pour la } \\
\text { liberté. Ce pays et le nôtre sont géographiquement éloignés, } \\
\text { mais beaucoup d'éléments de nos histoires se ressemblent. }\end{array}$ \\
\hline Petites difficultés d'existence & $\begin{array}{l}\text { Structure du roman (chaque chapitre porte le titre d'un } \\
\text { hexagramme de l'oracle chinois, le Yi King). } \\
\text { Langue parlée (le chiac de Moncton), anglicismes. }\end{array}$ \\
\hline Les Portes-tournantes & $\begin{array}{l}\text { L'histoire est pleine de sentiments positifs, d'une bonne } \\
\text { énergie. Ce qui m'a intéressée dès la première lecture, c'est la } \\
\text { pluralité des points de vue, l'histoire racontée par plusieurs } \\
\text { protagonistes. }\end{array}$ \\
\hline $\begin{array}{l}\text { Terre sur mer : « Clémence » } \\
\text { «a communion des } \\
\text { mélomanes" }\end{array}$ & $\begin{array}{l}\text { La narration en forme de rétrospection. La symbolique de la } \\
\text { mer et de la maison sur sable. } \\
\text { Le style de la langue parlée, les phrases courtes et les } \\
\text { relations temporelles. }\end{array}$ \\
\hline L'Oasis & $\begin{array}{l}\text { L'idée de voyager, de connaître de nouvelles cultures. Le } \\
\text { titre. }\end{array}$ \\
\hline $\begin{array}{l}\text { Disgrâce de l'Humanité : essai } \\
\text { sur la torture }\end{array}$ & $\begin{array}{l}\text { Grâce à l'auteur de cet essai, nous apprenons ce que c'est la } \\
\text { torture, quelle est « son histoire, son abolition, sa réapparition } \\
\text { au XXe siècle », ce qu'elle vole à un être humain. } \\
\text { Les différents intertextes, la nomenclature du tortionnaire. }\end{array}$ \\
\hline
\end{tabular}

\section{Annexe II : Barrières de compréhension}

\begin{tabular}{|l|l|}
\hline L'Acadien reprend son pays & $\begin{array}{l}\text { La méconnaissance de l'histoire acadienne est brouillée par le } \\
\text { fait qu'il s'agit d'un roman d'anticipation écrit dans les } \\
\text { années } 1970 \text { et dont l'action se déroule en 1987. Par } \\
\text { conséquent, il est difficile de distinguer ce qui appartient à la } \\
\text { réalité de ce qui est issu de l'imagination de l'auteur, p. ex. en } \\
\text { ce qui concerne les noms géographiques, les institutions. }\end{array}$ \\
\hline Petites difficultés d'existence & $\begin{array}{l}\text { Le chiac de Moncton et certains éléments concernant la vie } \\
\text { acadienne. L'oracle le Yi King. }\end{array}$ \\
\hline Les Portes-tournantes & $\begin{array}{l}\text { Certaines expressions toutes faites et des métaphores, p. ex. } \\
\text { - J'étais comme un petit soldat de bois qu'on remonte et qui }\end{array}$ \\
& $\begin{array}{l}\text { - tape le tambour jusqu'au bout du ressort. (29) } \\
\text { - Derrière lui, y a un tromboniste à la gorge profonde. (97) } \\
\text { - Concernant la conquête de Québec : } \\
\text { Un petit coup de Wolfe, un petit coup de Montcalm (...) (62) }\end{array}$ \\
& $\begin{array}{l}\text { Le langage se rapportant à la musique. } \\
\text { Ce qui m'a un peu frappée dans l'histoire présentée, c'est le } \\
\text { fait qu'Antoine qui a dix ans ne va pas (ou va très rarement) à } \\
\text { l'école et que ses parents ne réagissent pas à cette situation. }\end{array}$ \\
\hline Changement fréquent des temps verbaux, manque de \\
\hline Terre sur mer : «Clémence $»$
\end{tabular}

${ }^{9}$ Dans la colonne à droite je cite les fragments des réponses fournies par les étudiantes dans les enquêtes. 


\begin{tabular}{|l|l|}
\hline «La communion des & $\begin{array}{l}\text { relations cause-conséquence, les pensées inachevées ou les } \\
\text { sauts de pensée. } \\
\text { L'idée de la personnification de la maison sur le sable } \\
\text { paraissait exotique : «une drôle d'histoire ». }\end{array}$ \\
\hline L'Oasis & $\begin{array}{l}\text { Le fait que toute l'histoire est racontée à la troisième } \\
\text { personne, bien qu'il s'agisse des impressions de l'auteur. } \\
\text { Discours indirect libre (DIL). } \\
\text { La culture exotique de l'Inde : danses, coutumes, descriptions } \\
\text { de villes. }\end{array}$ \\
\hline sisgrâce de l'Humanité : essai & $\begin{array}{l}\text { Nomenclature juridique; techniques de la torture. } \\
\text { Les termes tels que le gêne, l'estrapade de terre, l'abbaye-de- } \\
\text { monte-à-regret (50). } \\
\text { Manque de traductions polonaises de certains documents } \\
\text { évoqués. } \\
\text { Le contenu de cet ouvrage peut être choquant, même, à } \\
\text { certains moments, obscène. Cela peut dégoûter le lecteur. } \\
\text { Pendant la première lecture de l'essai, les euphémismes } \\
\text { décrits dans le troisième chapitre intitulé "Comme un } \\
\text { lexique des abîmes » (50-60) surprennent immédiatement, p. } \\
\text { ex., M. le Tourmenteur-juré, Charlot-casse-bras. }\end{array}$ \\
\hline
\end{tabular}

Annexe III : L'universel

\begin{tabular}{|l|l|}
\hline L'Acadien reprend son pays & $\begin{array}{l}\text { La lutte pour l'indépendance, l'identité nationale. } \\
\text { Les Polonais connaissent le sentiment d'infériorité, ont subi la } \\
\text { domination des autres; les idées de liberté sont } \\
\text { particulièrement proches, mais aussi la famille et la tradition. }\end{array}$ \\
\hline Petites difficultés d'existence & $\begin{array}{l}\text { La vie quotidienne des gens : leur travail, le déménagement, } \\
\text { les difficultés de tous les jours. Aussi le fait de prévoir } \\
\text { l'avenir (tarot, horoscopes). } \\
\text { Protection, défense de sa langue. }\end{array}$ \\
\hline $\begin{array}{l}\text { Les Portes-tournantes } \\
\text { L'importance de la vie familiale : on peut observer des } \\
\text { parallélismes entre l'enfance d'Antoine et celle de son père et } \\
\text { dans les relations parents-enfants dans les différentes } \\
\text { générations. } \\
\text { Les allusions à la guerre. Comme la tragédie de la guerre joue } \\
\text { un rôle très important dans l'histoire polonaise, les Polonais } \\
\text { vont peut-être mieux comprendre le drame de Céleste. }\end{array}$ \\
\hline $\begin{array}{l}\text { Terre sur mer } \\
\text { «lémence » } \\
\text { «La communion } \\
\text { mélomanes » }\end{array}$ \\
$\begin{array}{l}\text { Les idées universelles : tromperie, jalousie, impossibilité de } \\
\text { résoudre les conflits. Valeur de sa propre maison, volonté de } \\
\text { fuir, de partir. } \\
\text { La tragédie des accidents de la route dus à l'abus de l'alcool. } \\
\text { La douleur éprouvée par les proches de ceux qui sont morts. } \\
\text { Il n'y a pas d'éléments particulièrement proches pour les } \\
\text { Polonais, mais cette problématique les concerne aussi. }\end{array}$ \\
\hline L'idée de voyager. \\
\hline L'Oasis
\end{tabular}




\begin{tabular}{|c|c|}
\hline $\begin{array}{l}\text { Disgrâce de l'Humanité : essai } \\
\text { sur la torture }\end{array}$ & $\begin{array}{l}\text { Le problème de l'existence de la torture dans le monde } \\
\text { contemporain devrait concerner tout le monde. On fait } \\
\text { référence à beaucoup de textes philosophiques, littéraires, au } \\
\text { droit international, aux conventions. } \\
\text { Les Polonais connaissent la torture de la Seconde Guerre } \\
\text { mondiale et de l'époque du stalinisme. Ils sont sensibles à la } \\
\text { discrimination. }\end{array}$ \\
\hline
\end{tabular}

\section{Annexe IV : Difficultés traductologiques}

\begin{tabular}{|c|c|}
\hline$L^{\prime}$ Acadien reprend son pays & $\begin{array}{l}\text { Informations concernant certaines connotations, p. ex. } \\
\text { Halifax - ville qui, dans l'histoire de l'Acadie, représente } \\
\text { la domination coloniale britannique. } \\
\text { Grand Dérangement } \\
\text { Aide à distinguer les évènements réels des évènements fictifs, } \\
\text { p. ex. } \\
\text { Dans les années 1980, pendant un hiver de famine, un } \\
\text { groupe de citoyens avait fait sauter le vétuste pont } \\
\text { ferroviaire de Madran. C'est ainsi qu'ils avaient arrêté un } \\
\text { train de victuailles destinées aux fêtes commémorant la } \\
\text { fondation d'Halifax. } \\
\text { 1755 - déportation des Acadiens (connotations : } \\
\text { souffrance, nostalgie) } \\
\mathbf{1 5} \text { novembre } \mathbf{1 9 7 6}-\text { commencement de la lutte pour } \\
\text { l'indépendance du Québec } \\
\text { Au niveau linguistique, certaines expressions, p. ex., le temps } \\
\text { de graines (c'est le moment de l'année quand les petites baies } \\
\text { et les fruits sont prêts à être récoltés). } \\
\text { Certains noms propres désignant les institutions, les cafés, le } \\
\text { nom des journaux, p. ex. } \\
\text { Commission Scolaire (l'administration locale dont } \\
\text { relèvent les écoles élémentaires et secondaires au Québec) } \\
\text { Évangéline - nom d'un journal acadien }\end{array}$ \\
\hline Petites difficultés d'existence & $\begin{array}{l}\text { Eléments culturels et realia, p. ex., } \\
\text { Restaurants - Tim Horton's, Osmose, Vieux Carré de la } \\
\text { Nouvelle-Orléans ; ensemble musical - Les Paiens, La } \\
\text { Salvation Army- magasin de journaux - Reid's ; } \\
\text { photographe de Moncton - Mathieu Léger ; villes - } \\
\text { Shédiac, Halifax, Barachois. } \\
\text { L'information concernant l'âge de maturité (19 ans au } \\
\text { Nouveau-Brunswick, mais } 18 \text { ans au Québec). } \\
\text { Dialogues en chiac, p. ex., } \\
\text { «Me semble qu'y'a trop de quoi qui va» (64): «il y trop de } \\
\text { choses qui se passent en même temps " ", } \\
\text { «Quoi c'qu'y va on... » (86): « qu'est-ce qui se passe», }\end{array}$ \\
\hline
\end{tabular}

\footnotetext{
${ }^{10}$ Nous traduisons en français standard.
} 


\begin{tabular}{|c|c|}
\hline & $\begin{array}{l}\text { "À Barachois, ben après-midi j'étais sûre qu'on allait flyer sus } \\
\text { l'île, de la manière que le vent était viré » (112): «Lisa est } \\
\text { arrivée à Barachois, mais sur la route cet après-midi, on } \\
\text { aurait cru que le vent qui soufflait très fort aurait pu les } \\
\text { pousser jusqu'à l'Île-du-Prince-Édouard » } \\
\text { "Yelle fait pas exactly zire non plus » (128) : «on ne peut pas } \\
\text { nier la beauté de Ludmilla Zablonski » } \\
\text { «On va aller ouère de quoi » (154): «on va aller voir quelque } \\
\text { chose ». }\end{array}$ \\
\hline Les Portes-tournantes & $\begin{array}{l}\text { Éléments culturels: } \\
\text { La bataille entre Wolfe et Montcolm } \\
\text { La Plaine d'Abraham } \\
\text { Éléments linguistiques (régionalismes) : } \\
\text { «l'air du temps » }(64) \text {; «ça lui fait toujours un petit velours » } \\
\quad(74) \text {; «ça sent la boule à mites » }(24) \text {; «je n'y vais pas par } \\
\text { quatre chemins» }(68)\end{array}$ \\
\hline $\begin{array}{l}\text { Terre sur mer : «Clémence » } \\
\text { «La communion des } \\
\text { mélomanes » }\end{array}$ & $\begin{array}{l}\text { Problèmes linguistiques: "Je dois y aller, je dois passer à la } \\
\text { commission ", "Acadian yuppies play traditional card games?", } \\
\text { "Le café_ jumbo », "régates internationales de Cocagne », } \\
\text { "Canadian Tire» (page), "Century } 21 », \text { "Central Real Estate», } \\
\text { «K-mart», etc. } \\
\text { Les consultations concernaient aussi les connotations } \\
\text { possibles de certains noms propres. }\end{array}$ \\
\hline L'Oasis & $\begin{array}{l}\text { Comme il s'agit d'une réalité étrangère à la réalité acadienne, } \\
\text { le savoir de la correspondante était au même niveau que celui } \\
\text { de l'étudiante polonaise. }\end{array}$ \\
\hline $\begin{array}{l}\text { Disgrâce de l'Humanité : essai } \\
\text { sur la torture }\end{array}$ & $\begin{array}{l}\text { Les consultations ont aidé à rassembler les informations sur } \\
\text { l'auteur et sur l'importance de l'œuvre au Canada. Pour } \\
\text { résoudre les difficultés traductologiques il a fallu consulter } \\
\text { d'autres sources. }\end{array}$ \\
\hline
\end{tabular}

\section{Annexe V : Réception}

\begin{tabular}{|l|l|}
\hline L'Acadien reprend son pays & $\begin{array}{l}\text { Le lecteur polonais (LP) peut apprendre beaucoup sur } \\
\text { l'Acadie. Cependant un certain savoir préalable concernant la } \\
\text { situation de la langue française et les événements historiques } \\
\text { évoqués par l'auteur semble nécessaire pour comprendre le } \\
\text { roman. Le LP doit aussi disposer d'un savoir concernant le } \\
\text { contexte social et historique de la création de l'œuvre pour } \\
\text { comprendre qu'il s'agit d'un roman d'anticipation. } \\
\text { Le LP aura de la difficulté à comprendre l'importance de } \\
\text { certains rituels, p. ex., la pêche du homard qui est décrite } \\
\text { dans le roman comme une activité presque sacrée. En } \\
\text { général, la valeur de la mer. }\end{array}$ \\
\hline Petites difficultés d'existence & $\begin{array}{l}\text { La traduction n'a pas pu rendre compte du chiac, ni de } \\
\text { certains effets comiques qui résultent de son utilisation. La }\end{array}$ \\
\hline
\end{tabular}




\begin{tabular}{|c|c|}
\hline & $\begin{array}{l}\text { traduction n'a pas non plus pu rendre compte des } \\
\text { anglicismes. } \\
\text { Ce texte est sûrement, pour un lecteur polonais, la source de } \\
\text { certaines informations concernant la vie quotidienne en } \\
\text { Acadie urbaine du sud-est du Nouveau-Brunswick. }\end{array}$ \\
\hline Les Portes-tournantes & $\begin{array}{l}\text { La non-connaissance de la réalité canadienne ne va pas } \\
\text { empêcher la compréhension du roman. Celui-ci peut être } \\
\text { intéressant pour le lecteur polonais de plusieurs points de } \\
\text { vue (histoire du Québec, faits de culture, etc.) }\end{array}$ \\
\hline $\begin{array}{l}\text { Terre sur mer } \\
\text { "Clémence », } \\
\text { communion } \\
\text { mélomanes » }\end{array}$ & $\begin{array}{l}\text { Les idées paraissent universelles. Le lecteur peut s'identifier } \\
\text { avec les héros. Un élément spécifique, mais qui n'empêche } \\
\text { pas la compréhension, c'est la symbolique de la mer. }\end{array}$ \\
\hline L'Oasis & $\begin{array}{l}\text { Le texte parle de la culture de l'Inde dont la compréhension } \\
\text { peut être la même au Canada qu'en Pologne. Dans l'original } \\
\text { il y a certains jeux de mots qu'on n'a pas réussi à rendre en } \\
\text { polonais. } \\
\text { Mais, en général, je ne vois pas de grandes barrières de } \\
\text { compréhension. }\end{array}$ \\
\hline $\begin{array}{l}\text { Disgrâce de l'Humanité : essai } \\
\text { sur la torture }\end{array}$ & $\begin{array}{l}\text { Cet essai ne porte pas sur les faits de culture; on ne peut } \\
\text { parler d'un lecteur polonais ou français. Il fallait plutôt dire } \\
\text { qu'un lecteur devrait être capable de comprendre la situation } \\
\text { difficile des victimes des tortures. Il est nécessaire de se } \\
\text { rendre compte de l'authenticité de cet ouvrage. }\end{array}$ \\
\hline
\end{tabular}

